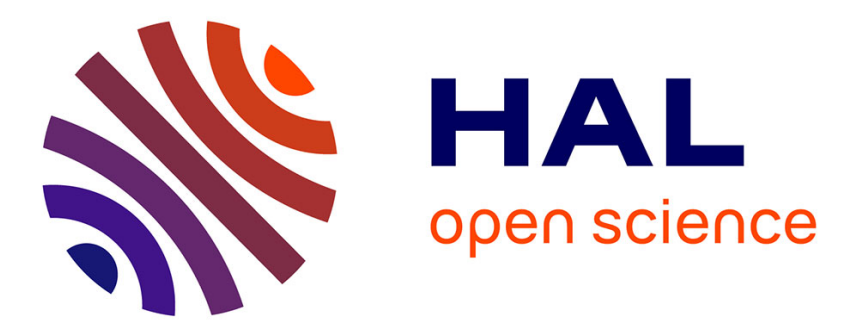

\title{
Traveling waves in a fluid layer subjected to a horizontal temperature gradient
}

\author{
François Daviaud, J.M. Vince
}

\section{To cite this version:}

François Daviaud, J.M. Vince. Traveling waves in a fluid layer subjected to a horizontal temperature gradient. Physical Review E : Statistical, Nonlinear, and Soft Matter Physics, 1993, 48, pp.4432-4436. 10.1103/PhysRevE.48.4432 . hal-00164453

\section{HAL Id: hal-00164453 \\ https://hal.science/hal-00164453}

Submitted on $20 \mathrm{Jul} 2007$

HAL is a multi-disciplinary open access archive for the deposit and dissemination of scientific research documents, whether they are published or not. The documents may come from teaching and research institutions in France or abroad, or from public or private research centers.
L'archive ouverte pluridisciplinaire HAL, est destinée au dépôt et à la diffusion de documents scientifiques de niveau recherche, publiés ou non, émanant des établissements d'enseignement et de recherche français ou étrangers, des laboratoires publics ou privés. 


\title{
Traveling Waves in a Fluid Layer \\ Submitted to a Horizontal Temperature Gradient
}

\author{
F. Daviaud and J.M. Vince \\ Service de Physique de l'Etat Condensé, CEA - Centre d'Etudes de Saclay \\ F-91191 Gif-sur-Yvette Cedex, France
}

\begin{abstract}
We report experimental observations of traveling waves in a pure fluid with a free surface situated in a long container submitted to a horizontal temperature gradient perpendicular to its large extension. Above a critical value of the gradient and depending on the height of liquid $h$, a source of waves is created in the container for small value of $h$, while the system exhibits stationary patterns for larger values of $h$. The spatio-temporal properties of the waves are studied and compared to theoretical predictions.
\end{abstract}


While the nature of turbulence in fluids is still today an open question, the evolution towards turbulent states has received much attention in the last decade and important results have been obtained in weakly confined systems. In this frame, the study of one-or two- dimensional (1D or 2D) systems in numerical models and in experiments has shown that a periodic cellular state may destabilize when a control parameter is varied and becomes turbulent through different scenarios. A transition to turbulence via spatio-temporal intermittency has been observed in models of phase equations [1] and coupled map lattices [2] as well as in 1D Rayleigh-Bénard convection [3], in the Printer's instability [4] or in capillary ripples [5]. A similar regime is also observed in circular [6] or plane Couette [7] flows. Spatio-temporal chaos can also appear by an increase of the number of defects as it is seen in Ginzburg Landau equations [8] or in convection in binary fluid mixtures [9]. Much work has been devoted to this last system in which the first state observed consists of traveling waves which arise from a subcritical Hopf bifurcation. This system displays a rich variety of dynamical states, such as confined states or defects and spatio-temporal chaos near onset in 1D [10] and 2D systems [11]. More recently, 1D propagative patterns have also been observed in a new experiment involving a hot wire below the free surface of a liquid. This dynamic state is qualitatively consistent with theories based on Ginzburg-Landau equations [12]. However, the physical mechanisms responsible for this instability remain unknown as well as the exact role of defects.

In this letter, we report new experimental results concerning different dynamical regimes, including traveling waves, obtained in a very simple configuration. A pure fluid with a free surface is situated in a long and narrow container and is submitted to a horizontal temperature gradient perpendicular to its large extension. Many experiments have been devoted to this problem, but always in containers with a small extension perpendicular to the gradient [13]. In this case, the propagating modes were never observed, whereas they can develop in our geometry. This problem has also received increasing attention in the past decade, because of microgravity facilities, in methods of float-zone crystal-growth with cylindrical geometries [14]. It was theoretically studied, in the case where gravity is ignored $[15,16,17]$. Our system thus appears to be a good tool to study both the mechanisms which drive this instability and the dynamics of nonlinear 1D traveling waves.

The apparatus consists of a rectangular container $20 \mathrm{~cm}$ long and $1 \mathrm{~cm}$ large (see Fig.1). The long vertical walls are made of copper and can be thermally regulated by circulating water. The container is closed by two small plexiglass walls. The lower boundary consists of a glass plate to allow visual observation while a plexiglass plate is inserted a few millimiters above the surface of the fluid 
to avoid evaporation problems. The container is filled with silicon oil (viscosity $\nu=0.0065$ Stokes) of Prandtl number $P=7$ up to a height $h$ that is measured with a precision of $0.05 \mathrm{~mm}$. The horizontal temperature gradient $\Delta T$ is imposed between the two copper walls and is measured using thermocouples. This gradient is regulated with a stability of $10^{-2} \mathrm{~K}$.

The patterns are visualized by shadowgraphic imaging. A parallel light beam crosses vertically the container from top to bottom and forms a horizontal picture on a screen, both due to surface deformations at the interface oil-air and to temperature gradients in the fluid (see Fig.2). The spatio-temporal evolution of the structures is recorded using a video camera and the images are digitized along a line of 512 pixels perpendicular to the gradient.

The two parameters which control our experimental system are $h$, the height of Si oil in the cell, and $\Delta T$, the horizontal temperature difference between the two walls. We have observed that, for each $h$ value, when $\Delta T$ is increased from zero to a threshold value $\Delta T_{c}$, a longitudinal (parallel to the gradient) pattern appears. But depending on the height of liquid, two different regimes take place. When $\Delta T \geq \Delta T_{c}$, for small $h$ values (from 0 to $2.8 \mathrm{~mm}$ ), the system exhibits longitudinal waves propagating perpendicularly to the gradient (see Fig.2) while, for larger $h$ values (from $2.8 \mathrm{~mm}$ to $10 \mathrm{~mm}$ ), stationary "rolls" with axis parallel to the gradient are observed. Figure 3 shows the dependence of $\Delta T_{c}$ on $h$ for these two kinds of structures. One can notice that the value of height $h=2.8 \mathrm{~mm}$ for which the two domains meet each other corresponds to a ratio between the Marangoni and the Rayleigh number $W=M a / R a \simeq 1$ with $M a=-(\partial \sigma / \partial T) \Delta T h / \rho_{0} \kappa \nu$ and $R a=$ $g \alpha \Delta T h^{3} / \kappa \nu$, where $g$ is the gravitational acceleration, $\alpha$ the thermal expansion coefficient, $\kappa$ the thermal diffusivity, $\rho_{0}$ the density of the fluid, $\nu$ the kinematic viscosity and $\sigma$ the surface tension. The traveling waves domain corresponds to $W>1$, which means that surface tension effects are dominant, while in the stationary rolls domain $(W<1)$, buoyancy is preponderant.

When studying the regime of propagative waves in more details, one can observe that the structures are not exactly perpendicular to the large side of the container but exhibit an angle $\psi \simeq 80^{\circ}$. This angle does not seem to depend on $\Delta T$ or on $h$. When $\Delta T$ is increased above $\Delta T_{c}$, a source of waves is created with a dimension which depends on $\varepsilon=\left(\Delta T-\Delta T_{c}\right) / \Delta T_{c}$. The size of the source is very large near the threshold and decreases in length as $\varepsilon$ is increased.

Space-time diagrams have been performed to study the dynamic of the waves (see Fig. 4). With such diagrams, the wavelength and the period of these waves can easily be measured. First of all, spatial and temporal Fourier spectra reveal that the system of waves has a unique wavelength and is monoperiodic at threshold. For $h$ values between 0 and $2.5 \mathrm{~mm}$, the period at threshold $T_{c}$ increases with $h$ (cf. 
Figure 5). In the same range of $h$, the $\lambda_{c} / h$ variation versus $h$ is no-monotonous, with a maximum for $h=1.4 \mathrm{~mm}$. These two behaviours can be compared to those obtained when a hot wire is used to destabilize a fluid layer with a free surface [12]. The physical context is somewhat different but the behaviour of the two sets of curves is very similar, allowing us to think that the physical mechanism which drives the instability in the hot wire experiment could have the same origin as in our configuration.

We have also performed some measurements for $\Delta T$ larger than $\Delta T_{c}$ at different $h$ values. The curves giving the period $T$ and the phase velocity $v_{\varphi}$ versus $\varepsilon$ are respectively displayed in Fig.6a and Fig.6b. The figures show that $T$ decreases and $v_{\varphi}$ increases when $\varepsilon$ is increased. One can notice that, for small $h$ values, the values of $T$ and $v_{\varphi}$ are clearly different from these obtained for the middle range of $h$ values. This fact shows how this phenomenon is sensitive to the surface tension effects which are more important as $h$ is small. Finally, when $\varepsilon$ is further increased, the system of traveling waves exhibit phase instabilities leading to space time dislocations.

For the stationary rolls, the experiment shows that they are strictly perpendicular to the large side of the cell, contrary to traveling waves. The wavelength associated to these rolls appears to be proportional to the height of liquid $h$. Then, when $\varepsilon$ is increased, the rolls begin to oscillate under the form of an optical mode, and, for larger $\varepsilon$, the pattern is destabilized and gives place to spatio-temporal chaos. These dynamical regimes are reminiscent of what is observed in RayleighBenard convection in narrow gap geometries [3].

Our observations raise several interesting issues concerning both the hydrodynamical and the phenomenological aspects. First of all, thermocapillarity appears to be the main physical mechanism driving the instability observed in our experiment for small values of $h$, while buoyancy is preponderant for larger values of $h$. It is known that, due to thermocapillary effects, fluid motion can develop when a temperature gradient is imposed on a thin fluid layer with a free surface [16]. Although we have not yet recorded velocity profiles, by seeding the flow with particles and illuminating it with a laser sheet, we have seen that the horizontal velocity profile reveals a shear flow near the surface and a return flow at the bottom, the convection being in the form of a monocellular cell (cf. [13]). Smith and Davis have shown that this dynamic state can destabilize under the form of two types of thermoconvective instabilities: stationary longitudinal rolls and traveling hydrothermal waves [15]. Some phenomena, such as temperature oscillations, had been previously observed in floating zones experiments [14], but, to our knowledge, our observations provide the first clear evidence of these instabilities. However, 
our experimental results share some differences with the results obtained by Smith and Davis. These authors consider the destabilization of two basic flow profiles: the linear flow state (LFS) and the return flow state (RFS). Stationary rolls are not stable in RFS but only in LFS. Moreover, oblique traveling waves are not predicted in fluids with $P>1$ in LFS while they do exist in RFS, but with an angle $\psi \simeq 20^{\circ}$ instead of $80^{\circ}$ as observed in the experiments.

These differences could be explained by the absence of gravity in their model. More recently, the stability of convective motion in a differentially heated cavity has been studied, in the presence of gravity $[18,19,20]$. However, certainly due to different boundary conditions, our experimental observations differ from their results. A more realistic model including both surface tension and gravity together with appropriate boundary conditions is at the moment studied and the first results are promising [21].

As for the phenomenological aspect, our configuration reveals to be a good system for exploring nonlinear 1D traveling waves and their destabilization. The spatio-temporal properties of the waves can be studied in the frame of two coupled amplitude equations, that could be derived from the physical model [21]. As a matter of fact, they have general properties that do not depend on the exact experimental system. In our experiement, the presence of a source stabilizes the right- and left-going waves with a definite wavelength and near threshold, the width of the source increases when decreasing $\varepsilon$, as it is observed in the hot wire experiment [12] and in recent numerical simulations [22]. Our experiment also shows similarities with some dynamical regimes (such as confined states with a stable sink of rolls) oberved in binary convection in an annulus $[9,10]$. We are performing experiments in this direction, using an annular configuration with a radial temperature gradient.

We wish to thank M.Dubois, J.Lega and C.Normand for stimulating discussions and M.Labouise, P. Hede and B. Ozenda for their technical assistance. 


\section{References}

[1] H.Chaté and P.Manneville Phys.Rev.Lett. 58, 112 (1987)

[2] K.Kaneko Prog.Theor.Phys. 74, 1033 (1985)

H.Chaté and P.Manneville, Physica D 32, 409 (1988)

[3] S.Ciliberto and P.Bigazzi, Phys.Rev.Lett. 60, 286 (1988)

F.Daviaud, M.Dubois and P.Bergé, Europhys.Lett. 9, 441 (1989)

[4] M.Rabaud, S.Michalland and Y.Couder, Phys.Rev.Lett. 64, 184 (1990)

[5] N.B.Tufillaro, R.Ramshankar and J.P.Gollub, Phys.Rev.Lett. 62, 422 (1989)

[6] C.D.Andereck, S.S.Liu and H.L.Swinney, J.Fluid Mech. 164, 155 (1986)

[7] F.Daviaud, J.Hegseth and P.Bergé, Phys.Rev.Lett. 69, 2511 (1992)

[8] P.Coullet and J.Lega, Europhys.Lett. 7, 511 (1988)

[9] see e.g. D.Bensimon, P.Kolodner, C.M.Surko, H.Williams and V.Croquette, J. Fluid Mech. 217, 441 (1990).

[10] P.Kolodner, J.A.Glazier and H.Williams, Phys.Rev.Lett. 65, 1579 (1990)

P.Kolodner, Phys.Rev. A 46, 6431 (1992)

[11] V.Steinberg, E.Moses and J.Fineberg, Nucl.Phys. B2, 109 (1987)

[12] J.M. Vince and M.Dubois, Europhys.Lett. 20, 505 (1992)

[13] see e.g. D.Villers and J.K.Platten, J. Fluid Mech. 234, 487 (1992).

[14] F.Preissier, D.Schwabe and A.Scharmann, J. Fluid Mech. 126, 545 (1983)

J.J.Xu and S.H.Davis, Phys.Fluids 27, 1102 (1984)

[15] M.K.Smith and S.H.Davis, J. Fluid Mech. 132, 119 (1983).

[16] S.H.Davis, Ann.Rev.Fluid Mech. 19, 403 (1987).

[17] M.K.Smith, J. Fluid Mech. 194, 391 (1988).

[18] P.Laure and B.Roux, J. Cristal Growth 97, 226 (1989)

[19] B.M.Carpenter and G.M.Homsy, J. Fluid Mech. 207, 121 (1989).

[20] G.Z. Gershuni, P.Laure, V.M.Myznikov, B.Roux, E.M.Zhukhovitsky, preprint submitted to Europ.J.Mech. B (Fluids)

[21] C.Normand and J.Lega, private communication

[22] F.Plaza, J.M.Vince and M.Dubois, private communication 


\section{Figure Captions}

Figure 1: Schematic drawing of the experimental apparatus.

Figure 2: Shadowgraphic image of a traveling wave pattern in an horizontal plane. The top (respectively the bottom) of the picture corresponds to the hot (repectively the cold) plate.

Figure 3: Critical temperature difference $\Delta T_{c}$ vs. the height of liquid $h$.

Figure 4: Spatio-temporal evolution showing a left- and a right going wave emitted from a source and corresponding to the pattern shown in Figure 2. The total acquisition time is $25 \mathrm{sec}$ and the spatial extension is $20 \mathrm{~cm}$.

Figure 5: Evolution of the period $T_{c}$ with the height of liquid $h$.

Figure 6: Evolution of the period $T$ (a) and of the velocity $v_{\varphi}$ (b) of the waves with the reduced temperature difference $\varepsilon$. 